$\begin{array}{ll}\text { Italique } & \text { Italique } \\ \text { Poésie italienne de la Renaissance }\end{array}$

XIX | 2016

Varia

\title{
Corrispondenza e corrispondenti nel secondo libro dei Sonetti di Benedetto Varchi
}

\section{Vanni Bramanti}

\section{(2) OpenEdition}

1 Journals

\section{Edizione digitale}

URL: http://journals.openedition.org/italique/431

DOI: 10.4000/italique.431

ISSN: 1663-4438

\section{Editore}

Librairie Droz

\section{Edizione cartacea}

Data di pubblicazione: 1 dicembre 2016

Paginazione: $87-112$

ISBN: 978-2-600-04777-7

ISSN: 1423-3983

\section{Notizia bibliografica digitale}

Vanni Bramanti, «Corrispondenza e corrispondenti nel secondo libro dei Sonetti di Benedetto Varchi », Italique [Online], XIX | 2016, online dal 01 décembre 2018, consultato il 06 septembre 2019. URL :

http://journals.openedition.org/italique/431 ; DOI : 10.4000/italique.431

(C) Tous droits réservés 


\author{
VAN I B RAMANTI \\ C ORR I S P O D E N Z A E \\ C O R R I S P O N D N T I N E L \\ SECONDO LIBRO DEI SONETTI \\ D I B E N E D E T T O V A R C H I
}





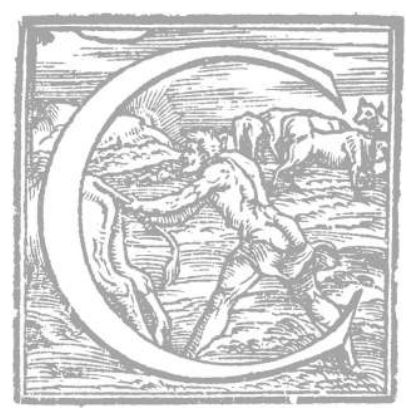

ome è emerso dagli studi succedutisi in questi ultimi decenni non esistono dubbi sull'importanza della figura e dell'opera di Benedetto Varchi all'interno degli anni centrali del nostro maturo Rinascimento, ${ }^{\mathrm{T}}$ non fosse altro per l'indubbia abilità nell' allacciare e sviluppare tutta una serie di rapporti con buona parte degli uomini di cultura del suo tempo. Senza niente togliere all'opera, coningata attraverso molteplici generi espressivi (con la punta di diamante della Storia fiorentina), la figura del personaggio presenta davvero risvolti di grande interesse: tanto nel corso del suo volontario esilio da Firenze (I537-I543), anni trascorsi quasi del tutto in area veneta con qualche puntata tra Bologna e Ferrara, quanto dopo il rientro nel capoluogo toscano (primi mesi del I543), Varchi non mancò di tenere costantemente aperta ed attiva la rete dei suoi contatti. Nelle molte dedicatorie dei suoi testi editi ed inediti, cosi come nelle purtroppo poche sue lettere delle quali a tutt'oggi siamo a conoscenza e in quelle che gli furono indirizzate nel corso del tempo, nel complesso, insomma, di questi materiali, è possibile rendersi conto della ariosa ricchezza del campionario di argomenti che gli stavano a cuore e che di volta in volta gli veniva fatto di condividere con $i$ suoi interlocutori. Oltre a tutto questo, ancora in merito a quanto appena detto, un ruolo primario bisognerà assegnare alla poesia di corrispondenza, nella sua interezza dispersa nelle tantissime carte manoscritte, ma che lo stesso Varchi ritenne opportuno fissare con la pubblicazione di due volumi: De' Sonetti di messer Benedetto Varchi, Firenze, Torrentino, I5ss (replica pressoché identica, anche se formalmente più corretta: Venezia, Pietrasanta, I555) e De' Sonetti di messer Benedetto Varchi a diversi e di diversi a lui. Parte seconda, Firenze, Torrentino, $1557,{ }^{2}$ in margine ai quali non sono mancati interventi significativi. ${ }^{3}$ Difficile stabilire con un minimo di precisione quando Varchi prese l'iniziativa di raccogliere nei due volumi citati una vasta silloge dei suoi sonetti di corrispondenza insieme a quelli che gli erano stati indirizzati, difficile, ripeto, a causa della latitanza di documenti specifici, a parte una lettera al Torrentino (in data 6 febbraio I555), nella quale, oltre a suggerire il titolo del primo dei due tomi, il diretto interessato non sembrava troppo soddisfatto del lavoro portato avanti in quei giorni 
dallo stampatore ducale. ${ }^{4}$ Inoltre, sempre in quel torno di tempo, Varchi «maturò la decisione, che sarà stata persuasa anche dalla misura, già grande e che sempre più s'ingrandiva, di separare l'unico libro canzoniere dall'epistolario poetico, che, visibile e definito, v'era stato racchiuso, e di dare a ognuno piena autonomia: l'uno pubblicato a stampa subito, l'altro due anni dopo».' Qualche altro elemento è possibile rintracciare nella lettera dedicatoria al principe Francesco de' Medici, all'inizio della quale si avvertiva l'illustre destinatario che, sulla traccia del Petrarca e del Bembo, Varchi svelava, pubblicando il primo volume delle sue rime, che avrebbe preferito non «doverle ad alcuna persona nominatamente intitolare»,, per poi aggiungere un'affermazione da non trascurare: «e spero di potere quando che sia, se non in maggior numero, certo con miglior forma stamparle». Pertanto, ormai davanti alle ultime prove di stampa, Varchi non mancava di sottolineare il suo scontento per quanto Torrentino stava facendo, avendo in queste sue recriminazioni perfettamente ragione, come del resto si può vedere scorrendo il volume, pieno di sciatterie, di errori, di sonetti collocati fuori posto, ${ }^{7}$ tanto è vero che in conclusione dell'Errata corrige, tra l'altro si legge:

Notisi, che i numeri delle faccie sono errati in moltissimi luoghi. Notisi, che i punti in alcuni luoghi mancano et in alcuni non stanno bene.

Forse lo stesso Varchi, o più probabilmente qualcuno che in sua vece stava seguendo a Firenze la stampa, con queste avvertenze intese in qualche modo anticipare le obiezioni che, stante la qualità del prodotto, sicuramente non potevano mancare. Inoltre, dall'intestazione della stessa lettera, "Orvieto, la vigilia del Corpusdomini dell"anno $M D L V »($ I2 giugno), non solo è possibile ricavare che a quell' altezza il libro non era venuto alla luce, ma anche che il relativo materiale era stato affidato al Torrentino già «più mesi», e che, infine, proprio gli ultimi passaggi prima della stampa stavano avvenendo senza la presenza dell'autore, allora ospite ad Orvieto di Lorenzo Lenzi, passaggi in merito ai quali, come abbiamo visto aveva avanzate le sue riserve lo stesso Varchi.

Tuttavia, prima di entrare nello specifico della presente ricerca, non sarà improprio soffermarsi almeno per qualche momento su alcuni aspetti della vita del Varchi nel periodo precedente alla pubblicazione dei suoi due libri di sonetti, cominciando con il ricordare che a poco più di tre 
anni dal suo ritorno a Firenze aveva ricevuto dal duca Cosimo de' Medici l'incarico ufficiale di scrivere la Storia fiorentina (I546). In particolare, a questa altezza cronologica, è noto il suo impegno con l'Accademia Fiorentina, onorato con molte lezioni e con l'assunzione della carica di console (marzo-settembre I545). Sappiamo anche che negli anni a venire, fatte salve alcune circostanze (tra le altre, la commemorazione funebre del Bembo e le due Lezzioni dedicate a Michelangelo), l'operosità varchiana all'interno di quello che allora era il maggior consesso culturale fiorentino, divenne sempre più impalpabile: chiamato ancora dal duca a commentare Petrarca (I553), agli inizi dell'anno seguente prese le distanze dall'Accademia, per infine tornarvi soltanto nell'estate del Is64 con due lezioni sull'amore in relazione a Purgatorio I7. Ricordato che, al tempo, ben poco venne pubblicato della cospicua attività accademia del Varchi, andrà comunque segnalato il suo lavoro di traduttore, il De consolatione philosophiae di Boezio (Firenze, Torrentino, I55I), testo al quale si dedicò dall'aprile del $1549^{9}$ e il De Beneficiis di Seneca (Firenze, Torrentino, I554), precedute nel 1548 dall'orazione pronunciata per la morte di Stefano Colonna e nel I549 dalla commemorazione funebre di Maria Salviati, madre del duca di Firenze. Se a tutto questo, mantenendo sullo sfondo la redazione della Storia, aggiungiamo che nel medesimo '49 si era occupato dell'edizione fiorentina (Torrentino) delle Prose del Bembo, sarà possibile, non lontano dal momento della preparazione dei due volumi di sonetti, rendersi conto della mole di lavoro che Varchi svolse nel corso di questo periodo, anche tenendo presente che non gli mancarono oneri che gli venivano dall'alto e che non era in grado di rifiutare, come nel caso delle tradurioni. Inoltre nello stesso anno di stampa del primo volume dei sonetti (I555), come già abbiamo visto, nell'estate Varchi fu ospite ad Orvieto di Lorenzo Lenzi, allora governatore di quella località, per poi raggiungerlo a Bologna, nel novembre, dove si era trasferito in qualità di vicelegato papale $;^{10}$ in seguito, nel I557, contemporaneamente alla pubblicazione del secondo tomo, a causa della grande esondazione dell'Arno avvenuta il 7 settembre, dopo qualche tempo fu costretto a trasferirsi a Pisa, ospite di Luca Martini, allora Provveditore dei Fossi di quella località. ${ }^{\mathrm{I}}$

A prescindere dalla tradizione rappresentata dalle antiche tenzoni, ormai nel cuore del secolo la poesia di corrispondenza non era certo una novità, dal momento che numerose testimonianze del genere possono 
essere con facilità rintracciate tanto nelle antologie liriche coeve, quanto nelle raccolte di singoli autori. Facendo riferimento ad alcuni significativi studi dedicati a questo fenomeno, ${ }^{\mathrm{I} 2}$ al di là, ripeto, di una forma espressiva che percorre senza soluzione di continuità più o meno l'intero secolo, non sarà male richiamare quanto consolidato dalla critica più attenta, e cioè la forte analogia tra le raccolte epistolari e la poesia di corrispondenza, generi, entrambi, che non a caso incontrarono un deciso consenso sul mercato librario del tempo:

Le affinità tra le lettere e le rime organizzate in forma collettiva sono allora profonde, poiché appartengono ad un medesimo clima culturale nel quale lo scambio e la condivisione del sapere è pratica fondamentale perché afferma e legittima il sistema stesso. ${ }^{13}$

Inoltre, ed anche questo è stato rilevato, nel caso del Varchi l'intero complesso della sua debordante produzione poetica appare connotato in modo assolutamente esplicito da un «carattere dialogico anche quando non è propriamente epistolare», ${ }^{\mathrm{I}}$ tanto è vero che la maggior parte dei sonetti compresi in quello che è stato definito il suo canzoniere amoroso (A) banno un preciso destinatario, ancorché non evidenziato in testa ai singoli componimenti, bensi proposto al lettore in un apposito Indice posizionato prima dei testi stessi e di nuovo, in chiusura, in una Tavola comprensiva di tutti gli incipit in ordine alfabetico, seguiti di volta in volta (con alcune eccezioni) dai nomi dei personaggi ai quali le rime venivano indirizzate. Anticipando quanto si vedrà più avanti, resta da aggiungere quello che forse rimane l'aspetto più evidente del passaggio da A a B, la sostanziosa riduzione operata dal Varchi nei confronti del gran numero di interlocutori che avevano affollato il suo primo libro di sonetti. Naturalmente in B compaiono non solo le proposte dell'autore insieme con le relative risposte, ma anche le proposte dei corrispondenti con le risposte dell'autore: si tratta, in definitiva, di un palese restringimento di campo, alludendo al quale si è addirittura parlato di «una sorta di elenco di affiliati a qualche segreta congrega»," 5 immagine volutamente eccessiva, ma grazie alla quale già da ora sarà possibile accennare ad una sorta di privilegiata mappa emotiva disegnata dal Varchi attraverso la struttura del suo secondo libro di sonetti, insieme alle esclusioni ed alle scelte operate rispetto al primo libro. Per inciso, come ben sanno quanti hanno avuto occasione di frequentare il maremagnum delle carte varchiane, non sembra superfluo aggiungere 
che il libro, nei dettagli del quale ci apprestiamo ad entrare, non risulta esaustivo dell'intera produzione poetica di corrispondenza dell'autore, visto che all'interno di quelle carte, oppure in stampe più o meno note, il genere di cui stiamo discorrendo appare largamente rappresentato. Di seguito almeno qualche esempio, a cominciare dai sonetti di scambio con Bernardino di Romena ${ }^{\mathrm{I}}$ conservati nelle citate Filze Rinuccini. Nella stessa BNCF altro interessante materiale si trova nel fondo Banco Rari: compresi nel plico LVIII, 2, una serie di sonetti scambiati tra Varchi e, tra gli altri, Bronzino, A. F. Grazzini, B. Davanzati; in LX, 4-I9 e 20-34, numerosi componimenti con L. Salviati; in LXIII, I7, l'interlocutore è Giovanbattista Adriani, con riferimenti alla redazione della Storia fiorentina. ${ }^{17}$ Ancora nel codice Magliabechiano, VII, 1388 (cc. 294 sgg.) sono compresi cinque sonetti indirizzati a Silvio Antoniano con le relative risposte, insieme a una dozzina di sonetti del Varchi al medesimo destinatario, questi senza repliche. Infine, a conclusione di un campione davvero di minima, i tanti sonetti a botta e risposta nella stampa settecentesca dei versi di Giovangirolamo de' Rossi. ${ }^{18}$ Venendo a questo punto ad un esame dettagliato di $\mathrm{B},{ }^{19}$ sarà bene cominciare dalla struttura del libro, che si apre con il seguente frontespizio: De' Sonetti di M. Benedetto Varchi colle risposte, e proposte di diversi Parte seconda, un titolo che rimanda immediatamente ad A, enfatizzando pertanto il collegamento con il primo dei due volumi tramite la dicitura Parte seconda, dando cosi ragione a quanti hanno colto la stretta contiguità tra le due raccolte. Dopo il frontespizio una semplice pagina bianca precede il primo sonetto (non a caso indirizzato al Caro), rispettando in tal modo quanto Varchi avrebbe voluto fare anche in $\mathrm{A}$, impostazione in tale circostan za del tutto appropriata, essendo l'opera interamente composta di testi rivolti a precisi interlocutori, fatto, questo, che finiva per escludere diper sél'esisten za di una dedica a unpersonaggio specifico. Dopo il ricordato sonetto (Caro, che nella dolce vostra, e acerba), la sequenza dei componimenti si succede senza soluzione di continuità: è il Varchi che imposta la proposta e i corrispondenti che gli rispondono, fino a che, con l'intervallo di un'altra pagina bianca ( $p .82)$, l'ordine si inverte, dal momento che ora sono i corrispondenti a proporre ed il Varchi a rispondere. Infine, le uniche forma di paratesto: Tavola de' Sonetti di M. Benedetto Varchi a diversi et di diversi a lui, cioè un indice dei sonetti disposti in ordine alfabetico con l'indicazione dei rispettivi destinatari e una Errata (Gli errori della stampa si correggono in 
questo modo), dove, a differenza di $\mathrm{A}$, non viene avanzata alcuna critica in merito alla qualità del lavoro dello stampatore. Come anticipato nel I557, data dipubblicazione di $\mathrm{B}$, Varchi era ormai da tempo lontano dalle attività dell'Accademia Fiorentina: l'anno prima, dopo aver trascorso alcuni mesi a Bologna, nel febbraio, tra l'altro inseguito dal Caro in vista dell'imminente polemica con il Castelvetro, ${ }^{20}$ era tornato a Firenze dove lo troviamo in corrispondenza con Ulisse Aldourandi, con ogni probabilità frequentato durante il citato soggiorno bolognese. ${ }^{2 \mathrm{I}}$ Altri interlocutori di questo periodo, Laura Battiferri Ammannati, impegnata a sollecitare pareri sulla sua produzione poetica ${ }^{22}$ e Giovan Battista Gelli, che già nel '43 si era attivato per il rientro a Firenze del Varchi al termine del suo volontario esilio, ${ }^{23}$ estensore in questa circostanza di una curiosa lettera nella quale appare come una sorta di consulente economico della famiglia del suo interlocutore. ${ }^{24}$ Ormai giunti al 1557 , sarà da ricordare che gran parte della prima metà dell'anno verrà trascorsa a San Gavino, con la cesura della già evocata esondazione dell'Arno del settembre, in seguito alla quale, anche se non nell'immediato, fu costretto a riparare a Pisa, dove giunse soltanto nei primi giorni di novembre. ${ }^{25}$ Essendo, dunque, B privo di ogni indicazione cronologica, alla luce dei dati appena riportati non appare possibile rendersi conto se e quanto Varchi abbia collaborato in occasione della stampa del secondo libro dei suoi sonetti; oppure, e anche questo sembra plausibile, Torrentino potrebbe aver ricevuto l'intero materiale tra la fine del I5s4 e i primi mesi del'ss e che, all'atto dell'andata in tipografia di $\mathrm{A}$, quanto riguardava $\mathrm{B}$ sia stato provvisoriamente accantonato. ${ }^{26}$ Ad avvalorare quest'ultima ipotesi il fatto che, almeno a mio avviso, non compaiono in $\mathrm{B}$ testi successivi al I554, data probabile di un sonetto del medico e poeta calabrese Giovanni Alfonso Mantegna (B, 179). ${ }^{27}$ Aggiungo che il volume raccoglie 409 sonetti, con 317 risposte per le rime e 92 per parole-rime. Nel complesso di 225 pagine, le proposte del Varchi sono comprese da p. 3 a p. 82; dopo due pagine bianche, le proposte degli interlocutori con le risposte del Varchi (pp. 85-225).

A questo punto qualche elemento di computisteria ci potrà aiutare a proposito del transito di corrispondenti tra $\mathrm{A} e \mathrm{~B}$, ricordando altresi che a meno di dieci anni dalla morte del Varchi videro la luce $i$ suoi Sonetti spirituali (Firenze, Giunti, I573), un libro ancora composto di sonetti, al cui interno, tra $i$ numerosi interlocutori, possiamo incontrare alcuni personaggi già presenti in $\mathrm{A} e$ in $\mathrm{B}$ e che vengono quindi 
a costituire una sorta di zoccolo duro dell'intera produzione poetica varchiana a stampa. In sostanza non più di quindici nominativi che merita comunque segnalare, accolti in due sezioni, amici-letterati e allievi. Nel primo gruppo figurano G. B. Busini, A. Allegretti, Bronzino, A. Caro, B. Cappello, F. Matteucci, G. B. Santini, B. Vechietti, G. Zoppio; nel secondo, L. Bonsi, G. della Stufa, L. Lenzi, A. Landi, L. Oradini. Fuori squadra, Francisco de Mendoza, cardinale di Burgos, frequentato dal Varchi durante il suo soggiorno fiorentino del maggio-novembre I553. A fronte di questo piccolo manipolo, ben 281 sono $i$ destinatari compresi in A per un totale di 534 sonetti; in $\mathrm{B}$, per un complesso di 220 sonetti (proposte e risposte), abbiamo IIo interlocutori: insomma, un calo netto rispetto al primo dei due volumi, un calo motivato da ragioni molteplici, a cominciare dal fatto che Varchi poteva non avere a disposizione $i$ relativi componimenti di risposta. Con maggior precisione, da A a B vengono meno 240 nominativi, una larga porzione di quell'universo al quale Varchi si era rivolto nel suo primo libro di versi, operazione, in verità, non semplice da decifrare, anche se, almeno in via di ipotesi, qualche connotato può venire rintracciato. In primo luogo non sono più rappresentati $i$ numerosi membri della famiglia Medici, gli Orsini (a parte l'«infiammato» Leone), Cibo, Farnese, Colonna e alcuni stretti collaboratori del duca Cosimo (L. Torelli, $F$. Mondragone), cosi come gli esponenti di antiche casate di fiorentine (Rucellai, Salviati, Soderini, eccetto Tommaso) e alcuni grandi del tempo (Carlo V con suo figlio Filippo, Garcia di Toledo), insieme a personaggi che in vario modo avevano condiviso il percorso del Varchi (Antonio da Barberino, A. Anselmi, A. Brucioli, M. Buonarroti, P. Carnesechi, Francesco di Vieri, D. Giannotti, G. Mazzuoli, J. Nardi, B. Segni, P. Vettori). Inoltre non resta traccia di vari nomi non fiorentini con $i$ quali era stato ed era tuttora in contatto (L. Boccadiferro, G. Cardano, M. Colonna, L. Contile, T. Gabriele, V. Gambara, G. Goselini, G. Guidiccioni, A. Lollio, P. Manuzio, F. Melchiori, G. Muzio, G. Ruscelli,S. Speroni, G. Stampa, C. Tolomei, G. G. Trissino, A. Vesalio). ${ }^{28}$

Ricordato che nel suo insieme B attesta IIo personaggi, da un ulteriore scrutinio risulta la sopravvivenza, sempre rispetto ad $\mathrm{A}$, di 40 nominativi, che, integrati come tra breve si vedrà dalle nuove entrate, costituiranno l'ossatura del libro. Al fine di stabilire almeno un certo 
ordine, si potrebbero ipotizare tre generose partiture, con la prima costituita da letterati e artisti, in qualche misura dal Varchi ritenuti amici: L. Alamanni, A. Allegretti, S. Bagnesi, Bronzino, G. B. Busini, A. Caro, G. Della Casa, F. del Migliore, S. Della Volta, M. Franzesi, Lodovico Martelli, Niccolò Martelli, Vincenzio Martelli, F. M. Molza, G. B. Pellegrini, G. B. Santini, B. Vecchietti. Lungo una direttrice che si dipana tra Venezia, Bologna e Napoli, sarà possibile identificare un secondo drappello: P. Bembo, B. Cappello, B. Ghezio, F. Matteucci, G. Moles, L. Orsini, D. Sandoval de Castro, L. Tansillo, F. Valentini, G. Zoppio. Infine, gli immancabili giovani fiorentini: B. Alamanni, P. Alberti, L. Bonsi, A. Del Bene, G. della Stufa, G. de' Rossi, A. Landi, L. Lenzi, U. Martelli, L. Oradini, C. Strozzi, M. Vivaldi. Anche se in misura nettamente minore nei confronti di $\mathrm{A}, i$ nomi appena evocati confermano l'orizzonte intellettuale ed emotivo del Varchi, che poteva contare su un ricca gamma di rapporti duraturied affidabili, che andavano da quella che era stata in assoluto la stella di una vita (Bembo) apresenze che avevano confortato i suoi trascorsi repubblicani, ad altri che avevano condiviso gli stessi sentieri della poesia (Alamanni, Cappello, Della Casa, itre Martelli, Molza, Tansillo), perfinire con gli scolari di un maestro senza cattedra che, al di là di ogni maldicenza, gli saranno costantemente affezionati e fedeli nel corso della sua intera esisten za.

Ancora sulla medesima falsariga, di nuovo qualche numero: all'interno di B emergono 70 nuovi personaggi, che, almeno in certa misura, rappresentano un coro di voci inedite, l'analisi delle quali, anche se parziale, può offrire più di un motivo di interesse, tanto più che, nell'economia del libro, siamo davanti a un numero assai alto, dal momento che a IIo ammontano $i$ personaggi nella loro totalità. Inoltre, come in precedenza annunciato, sostanziosa appare la riduzione degli interlocutori rispetto ad A, dove, ricordo, erano ben 28r: è ovvio che nel preparare il materiale per il volume Varchi fu condizionato da quanto in suo possesso, ricorrendo in larga misura a sonetti di scambio che aveva accumulato nel corso del tempo, anche se in realtà non mancano componimenti databili ad anni a ridosso della stampa del libro. Non pochi risultano gli autori deceduti, a cominciare da Lodovico Martelli, la cui morte sarà da situare tra la fine del I 27 e l'inizio del '28, Stiatta Bagnesi, scomparso in Puglia prima dell'assedio di Firenze $e^{29}$ e Francesco Berni (I535), che Varchi aveva avuto modo di incontrare a più riprese, in Mugello, dove il Berni era riparato dopo il sacco di Roma, a Bologna dove si trovavano entrambi per l'incoronazione di 
Carlo V, e negli ultimi anni di vita del poeta, stabilitosi dal I 333 a Firenze dopo il suo allontanamento dal cardinale Ippolito de' Medici. Nel corso del Is44 scompariranno sia il Molza che il Delminio, tutti e due frequentati dal Varchi nella secondaparte del'36 a Bologna, dove tra gli altri si trovava anche il Bembo, ${ }^{30}$ la cui morte cadrà nel' 47 , un anno dopo quella di Diego Sandoval de Castro, assassinato dai fratelli di Isabella di Morra, che aveva soggiornato a Firenze nei primi anni Quaranta, partecipe dell'Accademia degli Umidi e successivamente affiliato a quella Fiorentina. Nel Iss s sarà la volta di Vincenzio Martelli, che, lasciata Firenz̧e dopo la caduta della repubblica, nel I 30 era passato a Roma al servizio di Giovanni Gaddi, dove si trovava anche il Varchi, e poi a Napoli presso il principe Sanseverino. Più tardi, nel I563, lo stesso Varchi, insieme a Baccio Martelli, fratello del defunto, curò per i Giunti un'edizione delle sue rime. Quattro anni dopo, mentre A già si trovava nella bottega del Torrentino (forse insieme a B), si spense Niccolò Martelli, uno dei fondatori degli Umidi e molto vicino a Varchi nei lavori dell'Accademia Fiorentina. Nel Iss6 infine, a parte l'A retino con il quale ebbe rapporti sostanzialmente superficiali, vennero a mancare l'Alamanni, il Della Casa e Tullia d'Aragona, tutti e tre, al contrario, quanto mai importanti per la sua vicenda intellettuale ed umana.

Esaurito, almeno nei riferimenti essenziali, il paragrafo dei defunti, vedremo ora un altro aspetto significativo del libro, quello relativo ai personaggi incontrati da Varchi tra Padova e Venezia al tempo dell'Accademia degli Infiammati. Sottolineata l'imprevedibile assenza di Sperone Speroni (attestato, invece, in A, 104), ricordo i presenti in questo ambito, che appunto avevano avuto a che fare con il consesso padovano, a cominciare da Daniele Barbaro, accademico della prim'ora, e il patrizio veneziano Michele Barocci. ${ }^{3 \mathrm{I}}$ Di seguito, Bernardino Daniello, di cui sono noti $i$ rapporti con Varchi, ${ }^{32}$ il poeta genovese Emanuele Grimaldi, Leone Orsini, primo "principe" degli Infiammati, ${ }^{33}$ Alessandro Piccolomini, Antonio Maria Paccio, Francesco Sansovino, Bernardino Tomitano e Carlo Zancaruolo. Insomma, a circa tre lustri di distanza, includendo nel suo libro buona parte di quanti erano stati partecipi di quell'esperienza, Varchi, oltre che ribadirne la qualità all'interno del suo privato percorso, intese rendere omaggio a coloro che gli erano stati vicini nell'ultima parte del suo esilio. Ma non solo, perché nel giro degli Infiammati insieme a lui erano arrivati anche $i$ giovani che lo avevano seguito in terra veneta (Alberto 
del Bene, Lorenzo Lenzi, Ugolino Martelli e Carlo Strozzi), i versi dei quali troveremo puntualmente rappresentati in $\mathrm{B}$.

Proseguendo in questo succinto inventario, oltre ad autori largamente noti, alcuni dei quali già ricordati in precedenza (Alamanni, Aretino, Bembo, Berni, Caro, Della Casa, Delminio, Dolce, Domenichi, Grazzini, Molza, Tansillo, B.Tasso, Terracina), rimane un vasto territorio per gli esponenti della cultura e della società fiorentina (Adriani, Allegretti, Bagnesi, Bronzino, Busini, Fiamminghi, Franzesi, Landi, Nasi, Neroni, F e L. Sangallo, G. B. Strozzi, B. Vecchietti), ferma restando, come si è visto, la sostanziosa partecipazione degli "allievi", in margine alla quale sarà bene evidenz̧iare la quantità dei sonetti di risposta e di proposta afferenti a questi giovani (I4 di Lelio Bonsi, I3 di Lucio Oradini e 6 di Ugolino Martelli), ${ }^{34}$ sequenze di componimenti che venivano a costituire veri e propri nuclei all'interno della struttura del testo. ${ }^{35}$ A parte i rapporti familiari rappresentati nel volume da due nipoti, Antonio de' Bardi e Alessandro Menchi, ${ }^{36}$ compaiono alcuni collaboratori del duca di Firenze, da Sforza Almeni, a Filippo e Antonio del Migliore, a Pietro Gelido. ${ }^{37}$ Tra l'altro il citato Menchi non è il solo medico, vista la compagnia di colleghi a conferma del classico rapporto medicina-studi umanistici (Filippo Angeni, Antonio Graziosi, Pietro Medonio e Giovanbattista Pellegrini). ${ }^{38}$ Un altro significativo manipolo è costituito da alcuni personaggi attivi nella politica del tempo come Mario Bandini, Cristoforo Bellesanti, Antonio Gallo, Federico Lante e Gerolamo Mentovato, ${ }^{39}$ mentre le alte sfere del clero appaiono rappresentate da un solo esponente, Francisco Mendoza y Bobadilla, cardinale di Burgos. ${ }^{4 \circ}$ Ulteriori spunti di interesse, se non altro per misurare la portata fisica della corrispondenza varchiana, potrebbero scaturire da una ricognizione geografica dei luoghi in cui erano attivi $i$ tanti interlocutori, a questo fine ricordando che sarà proprio a Firenze che troveremo la netta maggioranza di questi ultimi, naturalmente comprendendo nel canone anche coloro che, per le più diverse ragioni, si trovavano lontani dalla loro terra d'origine oppure altri che, al contrario, in quella stessa città erano venuti a vivere. Subito dopo, quanti avevano condiviso con il Varchi i periodi della sua lontananza dalla patria, in particolar modo tra Padova e Venezia, come già abbiamo avuto modo di vedere: Bologna, località nella quale ebbe modo piu volte di trovarsi (Bolognetti, Mascherino, Pellegrini, Salvi, Zoppio), Modena (Bellesanti, Castelvetro, Molza, Valentini), Piacenza (Dome- 
nichi, in realtà frequentato a Firenze, Mentovato), Siena (Bandini, Piccolomini), Napoli (Mantegna, Moles, Rota, Serone, Tansillo), i lombardi Giovio jr. e Raineri, i marchigiani Alati, Gallo, Graziosi e Lante (altra cosa il Caro, di stanza a Roma), gli umbri Barbati e Benci, il viterbese Spira, il genovese Grimaldi. Anche se si tratta di un'esemplificazione non esaustiva, e dunque passibile di nuovi accessi, la mappa disegnata da Varchi attraverso $i$ suoi versi di proposta e le conseguenti risposte (e viceversa) finisce per coprire pressoché l'intero territorio della penisola: se al centro rimane la sua voce seduttrice, dai bordi del disegno si accampano tantissimi accenti che, al di là di ogni schema generazionale, tendono a raffigurare $i$ tratti di una ampia comunità che procede ben oltre le consorterie poetiche del tempo.

Da un esame sia pur parziale dei contenuti dei sonetti del Varchi sarà possibile avanzare qualche ipotesi sulle date di composizione dei medesimi, a partire da quello per Lodovico Martelli (B, 85), probabilmente il primo della serie, considerato il decesso del destinatario avvenuto tra la fine del I527 e l'inizio del I528. ${ }^{4 \mathrm{I}}$ Più o meno dello stesso periodo il componimento dedicato a Stiatta Bagnesi (B, Io), intimo del Varchi, come già ricordato venuto a mancare in Puglia poco prima dell'assedio di Firenze e noto per aver preso parte alla celebre edizione giuntina del Decameron pubblicata nel I527..$^{42}$ Ancora all'interno degli anni Trenta risalgono $i$ testi seguenti: nel Is34 troviamo un sonetto per l'allievo Ugolino Martelli in morte di Filippo Martini (B, I3), ${ }^{43}$ insieme ad un altri due del I536 (B, 89, 90) di risposta da Padova al giovane amico a Pescia, dove questi si trovava nell'autunno di quell'anno, ${ }^{44}$ al cui interno si parla della visita fatta al Bembo insieme a Lorenzo Lenzi, in margine alla quale nasce il componimento responsivo del Varchi $(\mathrm{B}, 172) .{ }^{45}$ Citato in precedenza il sonetto per il Berni, alla seconda parte del decennio (I537-38) risale una risposta in parolerima al Molza (B, I55), che, tra l'altro, ha dato adito ad una suggestiva proposta di interpretazione. ${ }^{46}$ Procedendo nel tempo, al febbraio del Is4I risale il sonetto indirizzato a Carlo Strozzi in morte di Giovanni Norchiati (B, I4), ${ }^{47}$ mentre al 1542 può essere assegnato il sonetto per Bernardo Sostegni (B, 36) grazie all'evocazione varchiana del suo «sospir trilustre», cioè $i$ quindici anni trascorsi dal giorno del suo innamoramento. ${ }^{48} \mathrm{Al}$ medesimo I542 appartiene il componimento destinato a Bernando Cappello (B, 78$)$, da non molto a Roma al servizio del cardinale Farnese, nella cui prima terzina viene celebrata la scom- 
parsa di Cola Bruno, avvenuta appunto in quell'anno; un altro sonetto di risposta allo stesso Cappello $(\mathrm{B}, 173)$ sembra risalire ad una data posteriore al I547, dal momento che mentre nella seconda terzina della proposta del Cappello viene evocato il Bembo che «infin dal ciel mi sgrida), nella risposta del Varchi una volta di più si sprecano gli elogi nei confronti di colui che era stato il suo «terrestre nume» e che «hor s'asside in mezzo a i piu perfettì. Al I544, e quindi dopo il rientro in patria del Varchi, risalgono $i$ due sonetti responsivi per Diego Sandoval de Castro (B, 2I6, 2I7), al tempo residente a Firenze e partecipe prima dell'Accademia degli Humidi e poi della Fiorentina. ${ }^{49}$ In precedenza già è stato ricordato il nome di Giovangirolamo de' Rossi, vescovo di Pavia, venendo ora ai due sonetti di risposta $(\mathrm{B}, 204,205)$ mi sembra plausibile, anche per una ragione di contenuto che vedremo più avanti, fissarne la datazione al I545, considerato che in questi versi auspicava per il suo interlocutore in volontario esilio in Francia un prossimo ritorno al suo «stato alto e preclaro», meglio se ammantato d'«alloro»e di petrarchesco ostro. ${ }^{50}$ Ormai ben noti $i$ rapporti con Bernardino Daniello, ${ }^{\mathrm{I}}$ di cui $(\mathrm{B}, 66)$ vengono ricordati sia il commento petrarchesco del Is4I (Venezia, Nicolini da Sabio) che le versioni virgiliane dell'undicesimo libro dell'Eneide e delle Georgiche del I545 (entrambe Venezia, Farri), riferimento, quest'ultimo, che ci consente di datare il sonetto. Al periodo successivo alla scomparsa del Bembo («al suo bel paese ritornato») rimanda una replica a Lodovico Domenichi $(\mathrm{B}, 185)$, cosi come un altro componimento, questa volta per Francesco Bolognetti $(\mathrm{B}, 47)$ dovrebbe riferirsi ad un lasso di tempo compreso tra il I547 e il '48, anno in cui il letterato bolognese portò a compimento Il Costante, opera a cui si riferiva il Varchi nella prima quartina del sonetto. Notato che $i$ due sonetti per Laura Terracina (B, $177-178)$ sono sicuramente anteriori al $1549,{ }^{52}$ già addentro agli anni Cinquanta, $\grave{e}$ possibile situare $i$ versi dedicati ad Antonio Allegretti (B, 82) al 1552 grazie al solito calcolo sullinnamoramento

Il quinto lustro ormai trapassa ed io

Già m'avvicino al cinquantesimo anno

Poi, che sempre entro e fuor senza alcun danno

Arsi Allegretto in casto foco e pio. ${ }^{53}$

Con ancora maggiore precisione sarà databile il sonetto per Lodovico Castelvetro (B, 5o), in quanto celebrativo della scomparsa di Gandolfo 
Porrino («che si gradito al coro / Fu delle Nove»), da Varchi incontrato a Firenze, insieme al Castelvetro, quando si trovava al seguito del cardinale Alessandro Farnese. ${ }^{54}$ Di nuovo i cinque lustri dalla data del colpo di fulmine ci permettono di situare nello stesso I5s2 anche il sonetto rivolto a Carlo Zancaruolo (B, 6I). ${ }^{55}$ Al successivo I553 appartengono con buona probabilità $i$ tre seguenti componimenti: il primo di risposta a Luigi Tansillo (B, I75), in quell'anno a Firenze al seguito delle truppe guidate da Pedro di Toledo, vicerè di Napoli, soggiorno nel corso del quale il poeta napoletano cadde gravemente ammalato ( «Ben del vostro languir tristo il Toscano / Paese duolsi» gli scriveva Varchi), condizione resa evidente nei versi del medesimo Tansillo ("Rotto dal corso in su'l terren Toscano / Di febbre, e di dolor mi struggo»). ${ }^{56}$ Il ricordato transito fiorentino di Francisco Mendoza consente di attribuire al ' 53 il sonetto a lui dedicato $(\mathrm{B}, 67)$, mentre un rimando all'età ("ch'io presso al cinquantesimo anno») situa nel medesimo torno di tempo una risposta a Michelangelo Vivaldi (B, I03). ${ }^{57}$ Ormai a ridosso della consegna al Torrentino del materiale da pubblicare in $\mathrm{A}$ (e forse anche di quanto verrà proposto in $\mathrm{B}$ ) risalgono almeno $i$ componimenti che ora saranno evocati, a cominciare da quello rivolto al napoletano Gabriele Moles (B, 52 ) dove si allude ai versi editi nel I5s4 in occasione del decesso di Maria Colonna. ${ }^{58}$ Ancora una volta il rimando all'innamoramento del Varchi ( $E$ E tale ad hora ad hor meco ragiona / Che sol ventisette anni in terra adoro») consente di situare nello stesso 's4 la data di un responsivo a Lorenzo Fiamminghi (B, 2I0); ${ }^{59}$ identico discorso per il sonetto indirizzato ad Antonio del Migliore (B, 73), nel quale Varchi ricordava il tempo, appunto ventisette anni prima, in cui le sue chiome «eran dorate e bionde». Infine, come ultimo esempio, il responsivo al poeta calabrese Giovanni Alfonso Mantegna (B, I79), anche questo, secondo un'ipotesi sostanzialmente verisimile, da attribuire al medesimo $1554 .{ }^{60}$

Al di là della presenza di temi e situazioni espressive inevitabilmente affini alla generica rimeria del tempo (le canoniche tappe dell'innamoramento, l'avanzare della vecchiaia con lo scontato corredo di capelli e barba imbiancati insieme a neve e gelo a profusione, il ricorso ai fumi evocati per identificare precisi luoghi geografici, la nostalgia per il tempo trascorso, l'esibita preferenza per una vita solitaria, meglio se inserita in ambito bucolico, e la conseguente avversione nei confronti del contesto 
urbano), ormai verso la conclusione del presente lavoro mette conto indugiare su un motivo ricorrente nei versi dei corrispondenti del Varchi, quello dell'invidia, della quale fu oggetto a più riprese. Già è stata ricordata la lezione che Varchi volle dedicare all'invidia, definita «rabbioso mostro e venenosissima peste», ${ }^{61}$ dove, oltre i consueti riferimenti letterari, sarà opportuno leggere più di un'allusione a quanto appunto l'invidia avesse gravato all'interno della sua privata vicenda. Tornato a Firenze nei primi mesi del I543, Varchi non aveva tardato sia ad entrare nelle grazie del duca Cosimo che nell'occupare una posizione di preminenza tra i membri dell'Accademia Fiorentina, fatti, questi, che, come è noto, gli procurarono pesanti inconvenienti, non solo nella cerchia intellettuale, quanto nel quotidiano svolgersi dell'esistenza, tanto è vero che dovette subire sia aggressioni verbali che attentati alle sua persona fisica, culminati nella primavera I545 quando fu incarcerato a causa di una supposta violenza esercitata su una giovanissima donna, violenza che secondo i suoi detrattori sarebbe avvenuta nel suo alloggio, circostanza nella quale vari personaggi di prestigio, primo fra tutti il Bembo, non esitarono a dimostrargli la loro solidarietà. ${ }^{62}$ A questo fine già sono stati segnalati $i$ sonetti del Bronzino (B, II6-II9), nel loro insieme svolti intorno al tema dellinvidia costantemente materializzatasi intorno alla persona del Varchi, ${ }^{63}$ con la sua opera innalzato «ove piu non si sale» $(\mathrm{B}, I I \sigma) e$ «Lunge dal volgo empio e fallace / Uso a fare a Miglior maggior oltraggio» $(\mathrm{B}, I I 7)$. Più decisamente calibrato sul tema si presenta un sonetto di Annibal Caro (B, I54) amico di lunga data, ${ }^{64}$ dove l'autore si rivolge in assoluta presa diretta alla «rabbiosa invidia», invano scagliatasi contro il Varchi che, da parte sua, risulta «puro, e saggio e pieno / D'ogni valore» e alla fine identificato in "Un nuovo Alcide, che per varij casi / Sofferendo e vincendo il ciel s'acquista». La prima quartina di un componimento di Trifone Benci $(\mathrm{B}, 196)^{65}$ offre un'ulteriore sopporto al presente discorso: «Assai dolor, ma poca meraviglia / Mi da Varchi il timor, ch'a voi sovrasta / Che sol virtù da invidia si contrasta», versi ai quali Varchi rispondeva con fermez?a, tra l'altro con una scoperta allusione al duca di Firenze, suo costante estimatore e protettore: «Ma io (mercè del Signor mio che fuora / Non uscio mai del dritto) assai beato / Non temo il verme, che i più rei divora». Anche nelle pieghe di un rapporto profondo quale fu quello tra Varchi e Tullia d'Aragona, attestato, tra l'altro, nella centralità del primo nel Dialogo della infinità di amore e dalle lettere da lei 
indirizzategli, ${ }^{66}$ il tema di cui ci stiamo occupando affiora in un sonetto della poetessa $(\mathrm{B}$, I99) dove, con il supporto degli abituali formulari, viene raffigurato il Varchi che, con la sua persona e con il suo «valor» finisce per colmare "'l mondo d'invidia». Sostanzialmente sulla stessa lungherza d'onda quanto sostenuto da Simone Della Volta, uno dei fondatori dell'Accademia degli Humidi, ${ }^{67}$ quanto mai esplicito nell'avvio del suo sonetto (B, I20):

Se molti, c'han d'invidia il rio cor pregno

Lacerando vi van più d'hora in hora

Pe i dolci frutti, e vaghi fior, che fuora

Produce il fertil vostro e colto ingegno

di nuovo insieme alla messa in rilievo del particolare rapporto esistente con il duca di Firenze ("Quanto il valor per cui v'ama et honora / Cosmo»). Sottolineata l'assidua presenza del Lasca nel corpo del volume, con la particolarità di una quartina $(\mathrm{B}, 93)$ che sembra disegnare una situazione ormai placata

Non si turbi hora il generoso core,

Però che 'l foco, che l'invidia accese,

È morto in tutto, e già vulgo scortese

Di se gl'incresce, e duolsi del suo errore

l'ultimo riferimento delle serie concerne il sonetto consolatorio di Anton Francesco Raineri (B, I98), composto in occasione della citata disavventura del marzo I545, dove una volta di più si esorta il Varchi a far di conto della protezione del "gran Cosmo». ${ }^{68}$ Da questi esempi contenuti nel numero, ma che avrebbero potuto facilmente essere di più, si possono ricavare almeno due osservazioni di ordine generale che vengono a proposito a conclusione del presente lavoro: in primo luogo si conferma quanto per altro già noto, cioè che Varchi, una volta rientrato nella città dalla quale si era volontariamente allontanato dopo l'omicidio del duca Alessandro de' Medici, non ebbe vita facile, trovandosi al centro di maldicenze e calunnie nella vita di ogni giorno e non di rado avversato nello svolgimento del suo impegno intellettuale, come appunto certificato dal suo contrastato percorso in seno all'Accademia Fiorentina. L'incarico attribuitogli dal duca Cosimo di scrivere la Storia fiorentina, proprio a lui che fino a quel momento (IS46) nella sua molteplice attività mai si era occupato di storiografia, dovette apparire a molti 
inopportuno, non da ultimo per la generosa e costante retribuzione ( I 50 scudi annui, in seguito portati a I80). Detto questo sarà immediato passare alla seconda delle due osservazione, la fondamentale importanza del medesimo Cosimo, non solo committente della Storia, ma, di fatto e come sappiamo, convinto protettore del Varchi, non solo ufficialmente provisionato ma anche sostenuto ed incoraggiato in piu di una circostanza. Infine, dall'intera esplorazione di $\mathrm{B}$, scaturisce un estremo codicillo: se Varchi a Firenze ebbe a trovarsi al cospetto di numerosi avversari, ma anche di affezionati e fedeli compagni, nei luoghi dove gli capitò di soggiornare durante il suo tutto particolare esilio non gli mancarono persone che avevano avuto modo di stimarlo e di ricordarlo per le sue qualità di letterato e di nomo.

Vanni Bramanti 
I. Per non appesantire una materia di per sé non troppo appetibile, mi limito a segnalare i contributi risultati davvero necessari ai fini della presente ricerca a cominciare da S. Lo Re, Politica e cultura nella Firenze cosimiana. Studi su Benedetto Varchi, Manziana, Vecchiarelli, 2008 e A. Andreoni, La via della dottrina. Le lezioni accademiche di Benedetto Varchi, Ets, Pisa, $20 \mathrm{I} 2$.

2. Da qui in avanti rispettivamente $A$ e $B$. Lasciando ad altri il compito di analizzare questi testi da un punto di vista formale, avverto che la mia attenzione sì è rivolta soprattutto ai corrispondenti del Varchi, in merito ai quali ho cercato di offrire qualche notizia sui personaggi meno conosciuti, senza pertanto soffermarmi su quelli largamente noti. Inoltre non ho ritenuto necessario proporre l'elenco completo dei corrispondenti in quanto già riportato in De Dante à Chiabrera. Poètes italiens de la Renaissance dans la bibliothèque de la Fondation Barbier-Muller. Catalogue établi par J. Balsamo con la collaboration de F. Tomasi, II, Genève, Fondation Barbier-Muller, 2007, pp. 25 I-52.

3. Da ricordare almeno B. Huss, "Cantai colmo di gioia, e senza inganni". Benedetto Varchis Sonetti (parte prima) im Kontext des italienischen Cinquecento-Petrarkismus", in «Romanistisches Jahrbuch», 52 (200I), pp. I 33-57; L. Paolino, Il 'geminato ardore' di Benedetto Varchi. Storia e costruzione di un canzoniere 'ellittico', in «Nuova Rivista di Letteratura Italiana», VII (2004), pp. 234-3 I 4; G. Tanturli, Una gestazione e un parto gemellare. La prima e la seconda parte dei Sonetti di Benedetto Varchi, in «Italique», VII (2004), pp. 45-100; D. Chiodo, Varchi rimatore. Modi e forme della poesia di corrispondenza, in Benedetto Varchi $1503-1565$, a cura di V. Bramanti, Roma, Edizioni di Storia e Letteratura, 2007, pp. I 57-7I.

4. Benedetto Varchi, Lettere (I535-1565), a cura di V. Bramanti, Roma, Edizioni di Storia e Letteratura, 2008, pp. I $52-53$.

5. Tanturli, Una gestazione, cit., p. 84.

6. A, p. 2.

7. Come già detto, sostanzialmente più corretta appare la coeva edizione di $A$ edita a Venezia dal Pietrasanta. Su questa stampa e in particolare sulla inaspettata dedica a monsignor Della Casa, cfr. Andreoni, La via della dottrina, cit., pp. 26-29. Le due edizioni vennero pubblicate a ridosso l'una dell'altra, infatti la dedica di quella fiorentina era del i 2 giugno, quella veneziana del i luglio.

8. Su tutto questo, Andreoni, La via della dottrina, cit., passim. Sulla situazione interna all'Accademia, resta fondamentale, M. Plaisance, L'Accademia e il suo Principe. Cultura e politica a Firenze al tempo di Cosimo I e di Francesco I de' Medici, Manziana, Vecchiarelli, 2004.

9. D. Brancato, Benedetto Varchi traduttore di Boezio, in Benedetto Varchi I503-I565, cit., pp. 96.

ıo. Agli inizi di dicembre Varchi si trovava infatti a Bologna, dove fu raggiunto da una lettera di Jacopo Nardi, cfr. Lettere a Benedetto Varchi (I530-I563), a cura di V. Bramanti, Manziana, Vecchiarelli, 20 I 2, pp. 346-47. 
I I. Fu soltanto nella primavera del i 558 che Varchi ritrovò una sistemazione stabile, quando il duca Cosimo, affinché potesse dedicarsi tranquillamente alla Storia, gli mise a disposizione la Topaia, una villa situata presso le residenze medicee della Petraia e di Castello, a pochi chilometri da Firenze. Nella ricordata alluvione Varchi, che alloggiava nei pressi del fiume, ebbe a subire la perdita di una parte notevole della sua cospicua biblioteca.

I 2. In particolare, l'introduzione di F. Tomasi all'edizione (San Mauro Torinese, Res, 2001) delle Rime diverse di molti eccellentissimi autori (Venezia, Giolito, I 545), curata insieme a P. Zaja; inoltre F. Tommasi, Alcuni aspetti delle antologie liriche del Cinquecento e P. Zaja, Intorno alle antologie. Testi e paratesti in alcune raccolte di liriche cinquecentesche, entrambi editi in I più vaghi e i più leggiadri fiori, a cura di M. Bianco e E. Strada, Alessandria, Edizioni dell'Orso, 200 I, pp. 77-I I e pp. I 33-45 (il saggio di Tomasi figura anche nei suoi Studi sulla poesia rinascimentale, Padova, Antenore, 20I 2, pp. 25-94).

I 3. Tomasi, Introduzione alle Rime diverse, cit., p. XI.

14. Chiodo, Varchi rimatore, cit., p. I 57.

I 5 . Chiodo, Varchi rimatore, cit., p. I 58.

I6. Cavaliere di Santo Stefano dal i 567 . I versi indirizzatigli dal Varchi in BNCF, Filze Rinuccini, 9, ins. I I, cc. 89-г г е г I, ins. 48, cc. 33 I-5 1. Ringrazio Dario Brancato per la segnalazione e ricordo che, a cura dello stesso Brancato, è in corso di completamento un inventario dei materiali varchiani compresi nelle Filže 3-16.

17. Su questi scambi di sonetti, mentre il presente contributo era in bozze, è stato pubblicato l'intervento di R. Cella, Sonetti di corrispondenza tra Benedetto Varchi e Lionardo Salviati (Firenze, Biblioteca Nazionale Centrale, Banco Rari 6o), in "Italianistica", XLV, 3, settembre-dicembre 2016, pp. 47-95.

18. Rime di M. Giovan Girolamo De’ Rossi, Bologna, Pisarri, I721, passim. Come sappiamo, a quanto sembra fu per primo il De' Rossi a comunicare al Varchi che il duca Cosimo voleva incaricarlo di scrivere la Storia fiorentina. Su questo significativo esponente della politica e della cultura negli anni centrali del secolo, si veda la Introduzione, redatta dallo scrivente, a Giovan Girolamo De’ Rossi, Vita di Federigo di Montefeltro, Firenze, Olschki, I 995.

I9. Volume in $8^{\circ}$, così come $A$.

20. Sul significato di questa celebre discussione cfr. S. Lo Re, Varchi tra Caro e Castelvetro, in Politica e cultura, cit., pp. 353-419.

2 I. VARCHI, Lettere I535-I565, p. I68 (lettera datata 28 febbraio I 556).

22. Lettere a Benedetto Varchi, cit., pp. 347-52 (alle pp. 348-49 essenziali riferimenti biobibliografici sul personaggio in questione).

23. Lettere a Benedetto Varchi, cit., pp. 222-26. 
24. Lettere a Benedetto Varchi, cit., p. 353. Lettera datata io febbraio i 557 e, come la precedente della Battiferri del 4 di febbraio, risulta indirizzata non a Firenze, ma alla pieve di San Gavino in Mugello. È ben noto che, sul piano culturale e all'interno dell'Accademia Fiorentina, il rapporto tra Gelli e Varchi non fu certo dei migliori.

25. «La lettera di vostra signoria mi è stata carissima per il desiderio ch'io havevo d'intendere della vostra giunta in Pisa sani e salvi» L. Battiferri, Maiano, 8 novembre I 557 , in Lettere a Benedetto Varchi, cit., p. 363.

26. Più o meno si tratta di quanto sostenuto in Tanturli, Una gestazione, cit., p. 84.

27. Ulteriori precisazioni più avanti. Avverto che, quando necessario, da qui in poi la sigla $B$ sarà seguita da un numero, relativo alla pagina della stampa, ricordando tuttavia che non sempre la numerazione originale risulta corretta.

28. Come vedremo, in $B$ verranno inclusi sonetti di risposta appartenenti ad autori deceduti, ovviamente ricevuti dal Varchi prima della loro scomparsa.

29. La notizia della morte del Bagnesi e del Martelli si trova nella biografia varchiana di G. B. Busini, ora in Lo Re, Politica e cultura, cit. p. I I s, dove entrambi vengono compresi tra $\mathrm{i}$ «primi amici» del biografato. Il Bagnesi, che aveva collaborato all'edizione giuntina del Decameron, è nominato dal Guicciardini nelle Storie fiorentine come «uomo di poca qualità» (Torino, Utet, I 99I, p. 21 8). Per quanto riguarda il Martelli, si ricordano le moderne edizioni della tragedia Tullia e delle Rime (Torino, Res, I 998 e 200 , rispettivamente a cura di F. Spera e L. Ammadeo).

30. Lo Re, Politica e cultura, cit., pp. I45-47.

3 I. Il Barocci (Barozio), già apprezzato dal Bembo per una sua orazione in lode della filosofia, figura, insieme a Daniele Barbaro, come protagonista del Dialogo della dignità delle donne dello Speroni, la cui casa era solito frequentare anche in compagni del Varchi (Bernardino Tomitano, Ragionamenti della lingua toscana, Venezia, Farri, I 545, p. I4).

32. Andreoni, La via della dottrina, cit., pp. 7 I-78.

33. Tra l'altro dedicatario del Discorso della bellezza e della grazia del Varchi (Andreoni, La via della dottrina, cit., p. 3 I 6 n.). Sotto il principato dell'Orsini, nell'estate del i 540, furono tenute dal Varchi tre lezioni petrarchesche (Rvf 29, Rvf I $82, R v f$ 2 I0).

34. Il Bonsi, laureato presso lo Studio di Pisa, fu accolto giovanissimo all'Accademia Fiorentina, diventandone successivamente Procuratore. Dopo la prematura scomparsa del cardinale Giovanni de' Medici, di cui era sottosegretario, entrò al servizio del principe Francesco e in seguito ricoprì la carica di Cancelliere dell'Ordine di Santo Stefano. Citato nell'Hercolano del Varchi, pubblicò le sue lezioni accademiche su Dante e Petrarca nel i 560 (Firenze, Giunti). Di origini perugine, Lucio Oradini fece stampare le sue lezioni nel i 550 (Firenze, Giunti); appare inoltre come dedicatario dello scritto del Varchi intitolato Delle vie delle 
dottrine e interlocutore di un Dialogo sopra la gramatica [...] dove s'introduce il Varchi e l'Oradino (Andreoni, La via della dottrina, cit., pp. I 5 I, 348-49). Il minor numero di sonetti del Martelli è probabilmente legato al fatto che, nel i 548, questi aveva lasciato Firenze per passare al servizio degli Strozzi, cfr. V. Bramanti, Ritratto di Ugolino Martelli, in «Schede umanistiche», 2 (I999), pp. 5-53.

35. Tanturli, Una gestazione, cit., p. 46-47 (altre serie tematiche qui individuate: «parentela dei corrispondenti», «casato», «invidia» che circondava il Varchi, argomento su cui si tornerà più avanti).

36. Il primo era figlio di Maria, sorella del Varchi, coniugata con Francesco de' Bardi; il secondo era un medico, molto vicino allo zio, con cui collaborò a più riprese. Insieme a lui, ed ad altri, giovani tra il dicembre del i 546 e il gennaio del I 547, alla pieve d San Gavino in Mugello, collazionò l'edizione aldina della Comedia dantesca (I I s) con sette codici antichi. Nel i 550 tenne presso l'Accademia Fiorentina una lezione su un sonetto del Bembo (Andreoni, La via della dottrina, cit., p. I 53). Inoltre si ha notizia che nel i 560 , insieme ad un altro medico (Francesco Catani), fu inviato a Ferrara per un consulto sulle condizioni di salute della duchessa Lucrezia, figlia del duca Cosimo e consorte di Alfonso II d'Este.

37. Il perugino Almeni a lungo maggiordomo principale del duca, nel i 566 fu da questi assassinato in seguito ad un inconsulto atto d'ira, Lettere a Benedetto Varchi (I530-I563), cit., p. 366 n. Dedicatario dei Cento sonetti raccolti dal Varchi per la morte di Luca Martini (Lo Re, Politica e cultura, cit., pp. 257-58), committente di numerosi artisti appare più volte nella Vita del Cellini (Benvenuto Cellini, Rime, a cura di D. Gamberini, Firenze, Sef, 2014, p. 96 n.). Filippo del Migliore (I $500-$ I 564) svolse prevalentemente la sua attività in orbita medicea: console dell'Accademia Fiorentina nel I 542 e nel I 5 2, a più riprese ambasciatore ducale, si dedicò in particolar modo alla ristrutturazione dello Studio pisano, di cui ricoprì la carica di Provveditore. Tra l'altro, nel I 543 fu tra quanti si attivarono per il ritorno a Firenze del Varchi (Lo Re, Politica e cultura, cit., pp. 29I-93). Antonio del Migliore, figlio di Filippo, fu anch'egli console dell'Accademia e Provveditore dello Studio, cfr. F. Tomasi, Odi ed elegie volgari di Benedetto Varchi, in Varchi e altro Rinascimento. Studi offerti a Vanni Bramanti, a cura di S. Lo Re e F. Tomasi, Manziana, Vecchiarelli, 2013 , p. I 80 . Piero Gelido, originario di San Miniato in provincia di Pisa, prima al servizio del cardinale Ippolito d'Este, si trasferi nel i 552 presso il duca di Firenze, dal quale, tra l'altro, venne inviato a Venezia (G. Dall'Olio, Diæionario biografico degli italiani, 53, pp. 2-5).

38. Accademico Fiorentino, l'Angeni fu espulso insieme al suo amico Antonfrancesco Grazzini ed altri in conseguenza della riforma dell'istituzione del i 546. Destinatario e personaggio di vari componimenti del Grazzini, in alcuni dei quali viene sbeffeggiato (ottava LX, capitolo XLI) e altrove definito «maestro e professor di medicina» (madrigalessa XXII). Originario di Montalboddo, oggi Ostra in provincia di Ancona, il Graziosi esercitò la professione a Terni; è inoltre ricordato per un trattato De rebus metereologis dedicato al duca Cosimo de' Medici. Il Medonio, forse originario di Ferarra, e il Pellegrini furono due medici attivi a Bologna. Due sonetti di quest'ultimo compaiono nella Raccolta in lode della illustrissima signora Donna Lucrezia Gonzaga (Bologna, s. e., I 565 , p. 68). 
39. Il senese Bandini (I 500-1 558) al tempo uno dei più importanti politici della sua città, tra l'altro podestà di Massa Marittima e ambasciatore presso Carlo V. Dedicatario dell'orazione De la prudenza di Bartolomeo Carli, Piccolomini fu in più occasioni vicino all'Accademia degli Intronati (F. Tomasi, L'Accademia degli Intronati e Alessandro Piccolomini: strategie culturali e itinerari biografici, in Alessandro Piccolomini (I508-1579). Un siennois à la croisée des genres et des savoirs, Paris, Sorbonne Nouvelle, Paris 3, 20 I I, pp. 25 n., 28 n.). Originario di Modena, il Bellesanti, fu intimo di altri corrispondenti modenesi del Varchi (Valentini, Castelvetro), dottore in legge, ricoprì significative cariche pubbliche nella sua città, prestando anche servizio presso il cardinale Morone (G. Mongini, Il racconto delle vite di alcuni letterati del suo tempo di Lodovico Castelvetro, in Lodovico Castelvetro. Letterati e grammatici nella crisi religiosa del Cinquecento, a cura di M. Firpo e M. Mongini, Firenze, Olschki, 2008, p. 343). L'urbinate Antonio Gallo, tra l'altro precettore di Francesco Maria II, ricoprì incarichi di prestigio alla corte ducale; ebbe altresì costanti interessi letterari, come attestato dalla sua presenza in vari epistolari (Ruscelli, B. Tasso) e dai suoi componimenti compresi in alcune raccolte (Cappello, Battiferri). Nel corso dei suoi studi a Urbino fu compagno di Torquato Tasso. Presente in alcune antologie poetiche del tempo (De le rime di diversi nobili poeti toscani, Bologna, Avanzo, I 565, p. 232 e Per donne romane. Rime di diversi, Bologna, Benacci, I 575 , p. 268), Federico Lante ricoprì, tra l'altro, carica di Gonfaloniere di Urbino. Il Mentovato, docente di Istituzioni allo Studio di Piacenza e uditore del duca Ottavio, e in seguito Governatore di Parma, insieme a Domenichi e a Doni fu uno dei più attivi membri della piacentina Accademia degli Ortolani. Compreso in varie edizioni delle Rime diverse, cfr. M. Baucia, Girolamo Mentuato. Una scheda, in «Bollettino storico piacentino», LXX, I ( 1980$)$, pp. 20 sgg.

40. Nel I 553 , in contrasto con Giulio III, il Mendoza, abbandonata Roma, era approdato a Firenze, dove soggiornò tra il maggio e dicembre. In questa circostanza fu frequentato dal Varchi, che lo accompagnò in un viaggio a Camaldoli e gli dedicò la lezione Sulla poetica in generale, esposta l'8 ottobre di quell'anno all'Accademia Fiorentina.

4I. In questa occasione Varchi dedicò un'elegia all'amico defunto (F. Tomasi, "Mie rime nuove non viste già mai ne toschi lidi". Odi ed elegie volgari di Benedetto Varchi, in Varchi e altro Rinascimento, cit., pp. 175-80). Il sonetto in questione già era apparso in Le rime volgari di Lodovico di Lorenzo Martelli (Roma, Blado, I 533 ), volume edito per iniziativa di Giovanni Gaddi, ora in Lodovico Martelli, Rime, a cura di L. Amaddeo, Torino, Res, 2005, p. ı o9. Sul Martelli cfr. P. Cosentino, Dizionario biografico degli italiani, 71, pp. 58-60.

42. Cfr. n. 30.

43. Fratello di Luca, il Martini morì nel settembre di quell'anno. Ricordato dal Varchi nell'egloga Dafne, cfr. Lo Re, Politica e cultura, cit., pp. 277-78.

44. Lo attesta una lettera del Martelli al Varchi compresa in Ugolino Marteldi, Lettere a Piero Vettori (I536-I577), a cura di V. Bramanti, Manziana, Vecchiarelli, 2009, pp. 3I-3I.

45. Edito più volte a partire dal i 542, ora in Pietro Bembo, Le rime, I, a cura di A. Donnini, Roma, Salerno, 2008, pp. 353-56. 
46. Chiodo, Varchi rimatore, cit., pp. I68-7I. Il componimento in questione è oggetto di un'analisi di F. Pignatti compresa in questo stesso fascicolo di «Italique».

47. Già si è incontrato lo Strozzi al seguito del Varchi, prima tra gli Infiammati a Padova e poi a Bologna. Rientrato a Firenze fece parte dell'Accademia Fiorentina per successivamente passare al servizio del cardinale Niccolò Ridolfi. Dopo aver preso parte alla guerra di Siena insieme ad altri fuorusciti si trasferì in Francia alla corte di Caterina de' Medici. Importanti e note alcune lettere scrittegli dal Varchi, raccolte in VARCHI, Lettere I535-I565, passim, che gli dedicò anche la sua Dichiaratione di tutti i termini principali e necessari della loica, cfr. Andreoni, La via della dottrina, cit., p. 52 e n. Il Norchiati, già membro dell'Accademia degli Humidi, aveva pubblicato un Trattato de' dipthongi toscani, Venezia, Sessa, I 539, cfr. F. Pignatti, Dizionario biografico degli Italiani, 78, pp. 73 I-34.

48. Il Varchi si innamorò del suo Lauro (Lorenzo Lenzi) verso la fine d'agosto del I 5 27, da lui conosciuto giovanissimo a Bivigliano durante un soggiorno presso i Della Stufa. All'interno di $B$ più volte si ricorre a questo espediente ben radicato nella tradizione lirica. Ricordo inoltre che il Sostegni, insieme a Luca Martini, appoggiò le ragioni del Varchi nel dibattito intorno al "verbo farneticare", cfr. Lo Re, Politica e cultura, cit., pp. 34I-43.

49. Già castellano di Cosenza il Sandoval aveva preso parte alla battaglia di Algeri. Durante un soggiorno a Roma aveva dato alle stampe le sue Rime (Dorico, I 542) ora, insieme a quelle di Isabella di Morra, Rime, a cura di T. R. Toscano, Roma Salerno, 2007: i sonetti del Varchi a pp. I40-4I. Fu ucciso nel i 546 dai fratelli della Morra, sua amante, cfr. T. R. Toscano, Un cavaliere inesistente: Don Diego Sandoval di Castro, in Letterati, Corti, Accademie: la letteratura a Napoli nella prima metà del Cinquecento, Napoli, Loffredo, 2000, pp. I 2 I-42.

50. Il de' Rossi rimase in Francia dal marzo al novembre del '45. Nell'aprile dell'anno successivo entrerà a far parte dell'Accademia Fiorentina, dove il 23 marzo di quell'anno il Varchi terrà una lezione sull'invidia a lui dedicata.

5 I. Andreoni, La via della dottrina, cit., pp. 7 I-78.

52. Sono infatti compresi nelle sue Rime seconde, Firenze, Torrentino, I 549, pp. 56,77 .

53. Ricordo che, come attestato nelle fonti biografiche, l'Allegretti era stato uno degli amici più stretti del Varchi, il quale, come è noto, attraverso di lui entrò al servizio della famiglia Gaddi. Astrologo e alchimista, autore della Trasmutatione de'metalli (ed. a cura di M. Gabriele, Roma, Edizioni Mediterranee, i98 I) e del poema Delle cose del cielo, che lo stesso Allegretti aveva affidato alla revisione del Varchi, cfr. Benvenuto Cellini, Rime, a cura di D. Gamberini, Firenze, Società Editrice Fiorentina, 20I4, p. 20.

54. Lodovico Castelvetro, Lettere Rime Carmina, a cura di E. Garavelli, Roma, Edizioni di Storia e Letteratura, 201 5 , pp. 22-23. Il Porrino era deceduto a Roma il 28 settembre I 552 . 
55. Corrispondente dell'Aretino, presente in varie raccolte di poesie del tempo, lo Zancaruolo è ricordato anche per la sua versione volgare del De' pesci romani di Paolo Giovio (Venezia, Gualtieri, I 560 ). Il suo sonetto di risposta al Varchi è ricordato in V. Merola, La fortuna del mito dell'Etna tra Cinquecento e Seicento, in www.academia.edu.

56. Questi e altri due sonetti tra Varchi e Tansillo appaiono recentemente editi in Luigi Tansillo, Rime, a cura di T. R. Toscano, Roma, Bulzoni, 20 i I, I, p. 380 ; II, pp. $779^{-80}$.

57. Ricordo che Varchi era nato a Firenze il ig marzo i 503. Poeta, tra i fondatori dell'Accademia degli Humidi e poi membro di quella Fiorentina, il Vivaldi è ricorrente nei versi del Grazzini e di Niccolò Martelli.

58. Le lagrime di Sebeto per la morte dellillustrissima Signora Donna Maria Colonna d'Aragona composte dal s. Gabriele Moles et nuovamente mandate in luce da Girolamo Ruscelli, Venezia, Griffio, I 54.

59. Di professione prete, il Fiamminghi era stato uno dei fondatori dell'Accademia degli Humidi. Noto in quanto autore di poesie burlesche, una sua canzone in morte di Alfonso de' Pazzi, detto L'Etrusco, figura in Rime burlesche di eccellenti autori, raccolte, ordinate e postillate da Pietro Fanfani, Firenze, Le Monnier, i 856 , pp. 94-96.

6o. È quanto sostenuto in Giovanni Alfonso Mantegna, Le rime, a cura di M. R. Bifolco, Salerno Edisud, 200 I, p. Io. Il Mantegna, originario di Maida in provincia di Catanzaro, si era trasferito a Napoli dove si era laureato in medicina, professione di fatto esercitata. Amico e corrispondente di Laura Terracina è ricordato inoltre come revisore delle edizioni dei versi della stessa.

61. Andreoni, La via della dottrina, cit., pp. I41-44.

62. "Gli amici suoi dall'altro canto parlorno al duca per lui, scrissono a Roma et per tutta Italia, procacciando da tutti i prelati et principi lettere favorevoli al duca per la salute sua», così nella biografia varchiana di Giovanbattista Busini, in Lo Re, Politica e cultura, cit, p. I I I.

63. Tanturli, Un parto gemellare, cit., p. 47.

64. Varchi e Caro diventarono amici dopo essersi incontrati nell'estate del i 527 a Bivigliano, sulle colline a settentrione di Firenze, entrambi ospiti nella villa della famiglia della Stufa.

65. L'intero scambio di sonetti con il Benci, vedrà la luce più tardi in De le rime di diversi nobili poeti toscani raccolte da Dionigi Atanagi, Venezia, Avanzi, I 565, pp. 5 Ir$54 \mathrm{r}$.

66. Il Dialogo di Tullia venne pubblicato nel I 547 (Venezia, Giolito), mentre la sua corrispondenza è compresa in Lettere a Benedetto Varchi, cit., passim. 


\section{VANNI BRAMANTI}

67. Inoltre, a più riprese il Della Volta appare nelle poesie di Antonfrancesco Grazzini, in particolare nella madrigalessa XL, dove viene evocato all'interno di una ideale compagna di amici, cfr. Le rime burlesche edite e inedite di Antonfrancesco Grazzini detto il Lasca, per cura di Carlo Verzone rinfrescate e nuovamente poste in luce da mastro Stoppino, Lulu, 201 5, p. 347.

68. Il componimento è riportato in Anton Francesco Raineri, Cento sonetti, a cura di R. Sodano, Torino, Res, 2004, p. 4I, a cui si rimanda anche per le notizie biografiche sul Raineri. 